\title{
Gastric wall fat falo sign as a risk factor for cardiovascular diseases
}

\author{
Ali Kupeli ${ }^{1}$, Ethem Unver ${ }^{2}$, Gurkan Danisan ${ }^{3}$, and Eser Bulut ${ }^{1}$ \\ ${ }^{1}$ Trabzon Kanuni Ĕ̆itim ve Araştırma Hastanesi \\ ${ }^{2}$ Erzincan Binali Yildirim Universitesi \\ ${ }^{3}$ Sakarya Universitesi
}

December 15, 2020

\begin{abstract}
A B S T R A C T Objective: To investigate the relationship between gastric wall fat halo sign and potentially associated cardiovascular disease (CVD) in thoracic computed tomography (CT). Material and Methods: Between October 2018 and June 2019, 62 patients with gastric wall fat halo sign and 97 controls were prospectively evaluated. Patient height, weight, body mass index (BMI), sex, age, ascending aorta, descending aorta, main pulmonary artery, right and left pulmonary artery, long and short cardiac axis and maximum transverse thorax diameters; and ascending, arcus, descending aorta and coronary artery calcium scores were recorded for the two groups. Results: No significant differences were found in sex, age, height, body weight or BMI between the two groups ( $\mathrm{p}>0.125$ ). Patients with gastric wall fat halo sign had significantly larger diameters of the ascending aorta, the descending aorta, the main pulmonary artery, the right and left pulmonary arteries, and the short and long cardiac axes and a higher cardiothoracic ratio (CTR) than the control group $(\mathrm{p}<0.001)$. Additionally, the calcium scores of the ascending, arcus, and descending aortas and the coronary arteries were significantly higher detected in patients group ( $\mathrm{p}$ $<0.001)$. Conclusion: The gastric wall fat halo is the result of excessive fat accumulation and can be observed in overweight people, especially those with increased visceral fat tissue. Additionally, patients with a gastric wall fat halo have a higher cardiovascular risk because of increased vascular diameters, CTR, heart sizes and calcium scores.
\end{abstract}

Gastric wall fat falo sign as a risk factor for cardiovascular diseases

\section{A B S T R A C T}

Objective: To investigate the relationship between gastric wall fat halo sign and potentially associated cardiovascular disease (CVD) in thoracic computed tomography (CT).

Material and Methods: Between October 2018 and June 2019, 62 patients with gastric wall fat halo sign and 97 controls were prospectively evaluated. Patient height, weight, body mass index (BMI), sex, age, ascending aorta, descending aorta, main pulmonary artery, right and left pulmonary artery, long and short cardiac axis and maximum transverse thorax diameters; and ascending, arcus, descending aorta and coronary artery calcium scores were recorded for the two groups.

Results: No significant differences were found in sex, age, height, body weight or BMI between the two groups ( $p>0.125)$. Patients with gastric wall fat halo sign had significantly larger diameters of the ascending aorta, the descending aorta, the main pulmonary artery, the right and left pulmonary arteries, and the short and long cardiac axes and a higher cardiothoracic ratio (CTR) than the control group ( $<<0.001)$. Additionally, the calcium scores of the ascending, arcus, and descending aortas and the coronary arteries were significantly higher detected in patients group $(\mathrm{p}<0.001)$. 
Conclusion: The gastric wall fat halo is the result of excessive fat accumulation and can be observed in overweight people, especially those with increased visceral fat tissue. Additionally, patients with a gastric wall fat halo have a higher cardiovascular risk because of increased vascular diameters, CTR, heart sizes and calcium scores.

Keywords: Cardiovascular disease, CT, gastric wall fat halo sign, obesity

\section{What is ready known about this topic?}

A fat halo sign refers to fat accumulation in the submucosal layer of the gastric, colonic and small bowel wall. The fat halo can also be commonly detected in overweight people, especially those with increased $v$ isceral fat tissue.

\section{What does this article add?}

Obesity is a remarkably heterogeneous condition, and $v$ isceral fat tissue has been revealed to be more deeply related to cardiovascular diseases than subcutaneous or total fat tissue. To the best of our knowledge, no study has investigated the gastric wall fat halo sign as a risk factor for cardiovascular diseases.

\section{INTRODUCTION}

Despite preventative and therapeutic efforts, cardiovascular diseases (CVDs), especially heart disease and stroke, are prominent causes of mortality and morbidity around the world ${ }^{1}$. The main risk factors and pathological mechanisms resulting in CVD can be traced back to childhood ${ }^{2}$. There are several controllable and uncontrollable risk factors for CVD, such as male sex, family history, old age, smoking, hypertension, physical inactivity, hypercholesterolemia, diabetes mellitus and obesity ${ }^{3}$.

The unfavorable consequences of overweight and obesity have increased worldwide and can be seen beginning in childhood ${ }^{4}$. Obesity is thought to be an independent risk factor for CVD, and increased body mass index (BMI) facilitates the development of comorbidities that contribute to $\mathrm{CVD}^{5,6}$. The generally preferred anthropometric technique to evaluate relative weight and classify obesity is BMI, which is the ratio of total body weight to height squared. Additionally, obesity is a remarkably heterogeneous condition, and $v$ isceral fat tissue has been revealed to be more deeply related to CVD than subcutaneous or total fat tissue ${ }^{7,8}$.

A fat halo sign refers to fat accumulation in the submucosal layer of the gastric colonic and small bowel wall $^{9}$. The fat halo in the intestinal wall was first associated with chronic inflammatory bowel diseases, but it can also be commonly detected in overweight people, especially those with increased $v$ isceral fat tissue $^{10,11}$. Additionally, fat halo sign can be observed in approximately $21 \%$ of patients who underwent computed tomography $(\mathrm{CT})$ examinations ${ }^{10}$.

There are only a few studies on gastric wall fat halo $\operatorname{sign}^{12,13}$. To the best of our knowledge, no study has investigated the gastric wall fat halo sign as a risk factor for CVD. In this study, we aimed to investigate the relationship between gastric wall fat halo sign and potentially associated CVD based on thorax CT.

\section{MATERIAL AND METHODS}

\section{Study Population}

Between October 2018 and June 2019, data from 71 patients who underwent unenhanced thorax CT and who had gastric wall fat halo sign were prospectively recorded. Three patients with chronic inflammatory bowel disease history and six patients with implanted coronary artery stents were not included in the study. Therefore, 62 patients were included in the final study.

For the control group, 97 patients with no gastric wall fat halo sign were selected from the clinical database of patients who underwent unenhanced thorax CT. Age, sex, height $(\mathrm{m})$ and body weight $(\mathrm{kg})$ were noted for the patient and control groups. Additionally, BMI $\left(\mathrm{kg} / \mathrm{m}^{2}\right)$ was calculated as the ratio of total body weight to height squared for both groups. This study was approved by the Institutional Ethics Committee.

CT Protocol 
The thorax CT examinations were performed with the participants in the supine position, and the patients were scanned from the lung apices to the adrenal gland level during a breath hold at the end of inspiration with using 16 slices (Siemens Somatom Sensation, Forchheim, Germany). All patients were examined using the standard scanning protocol without intravenous contrast agent. The CT protocol was as follows: 120 $\mathrm{kVp}$, tube current of 150-165 mAs, maximum $2.5 \mathrm{~mm}$ collimation, slice thickness of $1.5 \mathrm{~mm}$ and rotation time of $0.5 \mathrm{~s}$. Then, the images were reconstructed into multiplanar reformations.

\section{Analysis of CT Images}

The thorax CT images were evaluated by two radiologists (A.K. 8 years of thoracic radiology experience and M.K. 7 years of thoracic radiology experience) to reach a joint consensus. The images were transferred to the Syngo workstation (Siemens Medical Solutions). Linear fat accumulation in the submucosal layer of the gastric wall with attenuation of $<-10$ Hounsfield units (HUs) was described as a gastric wall fat halo sign (Figure 1). The measurements of the outer wall of the ascending aorta, the descending aorta, the main pulmonary artery, and the right and left pulmonary arteries were recorded in the axial plane at the level of the pulmonary artery bifurcation (Figure 2). After detecting the axial slice level that represented the maximum size of the heart, the long and short cardiac axes and the maximum transverse thoracic diameter were measured at the same level (Figure 3). Then, the cardiothoracic ratio (CTR) was calculated as the ratio of the long diameter of the cardiac axis to the maximum transverse thoracic diameter. The calcification of the ascending, arcus, and descending aortas and total coronary artery calcification were quantified by the method defined by Agatston et al. ${ }^{13}$. A region of interest (ROI) was positioned manually on the calcification of the ascending, arcus, and descending aortas and the coronary arteries. After selecting the total number of pixels over $130 \mathrm{HUs}$, the Agatston calcium score was computed with the software.

\section{Statistical Analysis}

All of the data were analyzed using the Statistical Package for the Social Sciences (SPSS 13.0 Statistical Software, SPSS Inc., Chicago, IL, USA) and the MedCalc package of Statistical Software version 16.8 (MedCalc Software bvba, Ostend, Belgium). Descriptive statistics, including the means and ranges, were calculated for age, height, BMI, CTR, the calcium scores of the ascending, arcus, and descending aortas and the coronary arteries, and the diameters of the ascending aorta, the descending aorta, the main pulmonary artery, the right and left pulmonary arteries, the short and long cardiac axes and the transverse thorax in the patient and control groups. The Kolmogorov-Smirnov test was used to identify deviations from anormal distribution. Additionally, the parametric Student's t-test was used to compare the age, height, BMI, and the diameters of the ascending aorta, the descending aorta, the main pulmonary artery, the right and left pulmonary arteries, the short and long cardiac axes and the transverse thorax. The nonparametric Mann-Whitney U test and the parametric Student's t-test were used to compare the calcium scores of the ascending, arcus, and descending aortas and the coronary arteries. A p value of less than 0.05 was considered to indicate a significant difference.

\section{RESULTS}

In this study, we analyzed 62 patients (45 male, 17 female) with gastric wall fat halo sign and 97 control patients (64 male, 33 female). The mean ages of the patient and control groups were $63.5 \pm 8.1$ and $62.1 \pm$ 8.8 years, respectively. The mean height $(\mathrm{m})$, body weight $(\mathrm{kg})$ and BMI $\left(\mathrm{kg} / \mathrm{m}^{2}\right)$ of the patient and control groups were $1.62 \pm 0.1 \mathrm{~m}$ and $1.63 \pm 0.1 \mathrm{~m} ; 80.8 \pm 13.4 \mathrm{~kg}$ and $77.4 \pm 13.7 \mathrm{~kg}$; and $30.6 \pm 4.9$ and $29.4 \pm$ $5.6 \mathrm{~kg} / \mathrm{m}^{2}$, respectively. There were no statistically significant differences in sex or the mean values of age, height, body weight or BMI between the two groups ( $\mathrm{p}>0.125)$.

The mean diameters of the ascending aorta, the descending aorta, the main pulmonary artery, the right and left pulmonary arteries, the short and long cardiac axes and the transverse thorax, the CTR, and the calcium scores of the ascending, arcus, and descending aortas and the coronary arteries are presented in Table 1. Patients with gastric wall fat halo sign had significantly larger diameters of the ascending aorta, the descending aorta, the main pulmonary artery, and the right and left pulmonary arteries than the control group $(\mathrm{p}<0.001)$. Although the short and long diameters of the cardiac axis and CTR were observed to 
be significantly higher in patients with gastric wall fat halo sign $(\mathrm{p}<0.001)$, no statistically significant difference was detected in the transverse thoracic diameter between the two groups $(\mathrm{p}=0.861)$. Additionally, the calcium scores of the ascending, arcus, and descending aortas and the total and coronary arteries were significantly higher in patients with gastric wall fat halo sign than in patients without a gastric wall fat halo sign $(\mathrm{p}<0.001)$ (Figure 4).

\section{DISCUSSION}

In the present study, we investigated the relationship between gastric wall fat halo sign detected by thorax CT and CVD. We found that gastric wall fat halo sign might be detected in patients with increased aorta and pulmonary artery diameters and calcium scores.

Although there are several studies of the fat halo sign in the gastrointestinal tract, there are few studies involving the fat halo sign in the gastric wall ${ }^{10-16}$. To the best of our knowledge, this is the first study investigating the relationship between gastric wall fat halo sign and associated CVD based on thorax CT.

The fat halo sign was first thought to indicate the presence of inflammatory bowel disease. However, fat halo sign can be seen especially in overweight people. Additionally, gastric wall fat halo sign can be observed in patients without gastrointestinal disease and with increased visceral fat tissue ${ }^{12,13}$. After fat is stored in normal adipose tissues, free fatty acids are discharged into the blood ${ }^{17}$. The fat halo sign could be related to an increase in the blood concentration of free fatty acids and could be an indicator of excessive fat accumulation $^{17}$.

The ascending and descending aorta diameters are easily evaluated through chest CT. In our study, we found that patients with gastric wall fat halo sign had significantly higher ascending and descending aorta diameters than those without fat halo sign. One of the causes of aortic diameter increase is visceral obesity. The increase in aortic diameter associated with visceral obesity could be related to the process of adaptation to hypertension, increased blood volume and underlying structural or functional aortic abnormalities ${ }^{18}$. Additionally, visceral obesity can stimulate vascular aging, resulting in future cardiovascular events ${ }^{8}$. Visceral obesity and gastric wall fat halo sign are associated with each other, so increased aortic diameters may be related to this relationship.

Although pulmonary hypertension may appear to be a sporadic disease with undetectable risk factors, it can occur as a result of preexisting medical conditions ${ }^{19}$. Pulmonary hypertension and obesity are generally two coexisting conditions in the clinic, and increased BMI induces the development of pulmonary hypertension ${ }^{20}$. Chan et al. reported that there were more obese participants in the pulmonary hypertension group than in the control group, whereas most patients were not obese in both groups ${ }^{21}$. In this study, we revealed that patients with gastric wall fat halo sign had significantly higher main, right and left pulmonary artery diameters than those without fat halo sign. This relationship may be the reason for pulmonary artery enlargement in our study.

Obesity is connected with most CVDs, such as coronary heart disease, hypertension and heart failure ${ }^{22}$. In particular, visceral adipose tissue is hormonally active tissue that contributes to vascular inflammation by producing adipokines and is a risk factor for mortality and $\mathrm{CVD}^{23}$. Additionally, obesity is related to some hemodynamic changes, such as increased cardiac output, diastolic filling pressure, left ventricle hypertrophy and ventricular dilatation ${ }^{24}$. The CTR is usually used to evaluate cardiomegaly in chest radiography, cardiomegaly indicating underlying CVD, is evidenced by hypertrophy or dilation of the heart ${ }^{25}$. CT imaging is a simple and effective method for assessing cardiomegaly. Despite a lack of significant difference in the transverse thoracic diameter, we found that patients with gastric wall fat halo sign had significantly higher short and long diameters of the cardiac axis and CTRs than the control group. These results may be related to cardiovascular changes caused by visceral adipose tissue.

Atherosclerosis is a major cause of death and morbidity in industrialized societies due to its association with cardiovascular events and peripheral vascular disease. Atherosclerosis has different stages, and calcification indicates advanced atherosclerotic lesions ${ }^{26}$. Atherosclerosis starts with fatty streaks and advances further to 
calcified plaques in adulthood. Although the cause of vascular calcification is not fully understood, inflammation, dysregulated metabolism, and osteogenesis are some processes associated with vascular calcification ${ }^{27}$. Thoracic aortic calcification represents a systemic atherosclerotic burden and results in an increased risk of cardiovascular events ${ }^{28}$. Visceral fat tissue is an endocrine organ that can produce atherogenic agents. Additionally, this condition can induce vascular aging resulting in an increased risk of CVDs, hypertension and calcified atherosclerotic plaques ${ }^{29}$. It has been reported that the risk of aortic atherosclerosis increases as the amount of visceral fat tissue increases ${ }^{30}$. Moreover, aortic wall calcification can be assessed with CT and lateral X-ray ${ }^{31}$. In our study, we used non contrast thoracic CT to evaluate thoracic aortic calcification and found that patients with gastric wall fat halo sign had significantly higher calcium scores in the ascending, arcus, and descending aortas than patients without fat halo sign.

Coronary artery disease, caused by atherosclerosis, is a main cause of morbidity and mortality worldwide, so the early diagnosis of subclinical atherosclerosis is crucial for preventing coronary artery disease. Similar to thoracic aortic calcification, obesity is an independent risk factor for atherosclerosis and coronary artery calcification $^{32}$. The calcium score of coronary arteries indicates the burden of atherosclerotic plaque in arteries and is a helpful, noninvasive method for the assessment of subclinical atherosclerosis in asymptomatic patients ${ }^{33}$. Additionally, the calcium score of coronary arteries is connected with cardiovascular events and obesity $^{33}$. In this study, we showed that higher calcium scores of coronary arteries were detected in patients with gastric wall fat halo sign related to visceral obesity.

This study has a number of limitations. First, the CT images were evaluated based on a consensus, and we did not evaluate the inter- or intraobserver variability in our study. The second limitation was that the number of patients was also relatively small. The third limitation was that participants in the patient and control groups did not undergo abdominal CT imaging, so visceral fat tissue measurement could not be performed in our study. Fourth, we did not have access to laboratory parameters such as lipid panels.

\section{CONCLUSION}

The gastric wall fat halo results from excessive fat accumulation and can be observed in overweight people, especially those with increased visceral fat tissue. Additionally, patients with a gastric wall fat halo have higher cardiovascular risk because of increased vascular diameters, CTR, heart sizes and calcium scores. However, extensive studies with larger populations are needed to clearly confirm the relationships of gastric wall fat halo sign with cardiovascular diseases.

\section{References}

1. Xu J, Murphy SL, Kochanek KD, Bastian BA. Deaths: final data for 2013. Natl Vital Stat Rep 2016; 64(2):1-119.

2. Steinberger J, Daniels SR. American Heart Association Atherosclerosis, Hypertension, and Obesity in the Young Committee (Council on Cardiovascular Disease in the Young); American Heart Association Diabetes Committee (Council on Nutrition, Physical Activity, and Metabolism). Obesity, insulin resistance, diabetes, and cardiovascular risk in children: an American Heart Association scientific statement from the Atherosclerosis, Hypertension, and Obesity in the Young Committee (Council on Cardiovascular Disease in the Young) and the Diabetes Committee (Council on Nutrition, Physical Activity, and Metabolism). Circulation 2003; 107(10):1448-53.

3. Fryar CD, Chen TC, Li X. Prevalence of uncontrolled risk factors for cardiovascular disease: United States, 1999-2010. NCHS Data Brief 2012; (103):1-8.

4. Flegal KM, Carroll MD, Ogden CL, Curtin LR. Prevalence and trends in obesity among US adults, 1999-2008. JAMA 2010; 303(3):235-41.

5. Poirier P, Giles TD, Bray GA, Hong Y, Stern JS, Pi-Sunyer FX, et al. Obesity and cardiovascular disease: pathophysiology, evaluation, and effect of weight loss. Arterioscler Thromb Vasc Biol 2006; 26(5):968-76. 
6. Ortega FB, Lavie CJ, Blair SN. Obesity and Cardiovascular Disease. Circ Res. 2016 May 27;118(11):175270 .

7. Owens S, Gutin B, Ferguson M, Allison J, Karp W, Le NA. Visceral adipose tissue and cardiovascular risk factors in obese children. J Pediatr 1998; 133(1):41-5.

8. Després JP. Cardiovascular disease under the influence of excess visceral fat. Crit Pathw Cardiol 2007; $6(2): 51-9$.

9. Wittenberg J, Harisinghani MG, Jhaveri K, Varghese J, Mueller PR. Algorithmic approach to CT diagnosis of the abnormal bowel wall.RadioGraphics 2002; 22(5):1093-107.

10. Harisinghani MG, Wittenberg J, Lee W, Chen S, Gutierrez AL, Mueller PR. Bowel wall fat halo sign in patients without intestinal disease. AJR 2003; 181(3):781-4.

11. Pickhardt PJ, Asher DB. Wall thickening of the gastric antrum as a normal finding: multidetector CT with cadaveric comparison. AJR 2003; 181(4):973-9.

12. Gervaise A, Naulet P, Gervaise-Henry C, Junca-Laplace C, Pernin M, Lapierre-Combes M. Gastric Wall Fatty Infiltration in Patients Without Overt Gastrointestinal Disease. AJR Am J Roentgenol 2016; 206(4):734-9.

13. Kupeli A, Danisan G, Kocak M, Taskent I, Balcı IG, Bulut E. Gastric wall fat halo sign in patients without intestinal disease. Clin Imaging 2019; 54:31-6.

14. Jones B, Fishman EK, Hamilton SR, Rubesin SE, Bayless TM, Cameron JC, Siegelman SS. Submucosal accumulation of fat in inflammatory bowel disease: CT/pathologic correlation. J Comput Assist Tomogr. 1986;10(5):759-63.

15. Ahualli J. The fat halo sign. Radiology 2007; 242(3):945-6.

16. Muldowney SM, Balfe DM, Hammerman A, Wick MR. Acute fat deposition in bowel wall submucosa: CT appearance. J Comput Assist Tomogr 1995; 19(3):390-3.

17. Sacks HS, Fain JN. Human epicardial adipose tissue: a review. Am Heart J 2007; 153(6):907-17.

18. Jayalath RW, Mangan SH, Golledge J. Aortic calcification. Eur J Vasc Endovasc Surg 2005; 30(5):476-88.

19. Summer R, Walsh K, Medoff BD. Obesity and pulmonary arterial hypertension: Is adiponectin the molecular link between these conditions? Pulm Circ 2011; 1(4):440-7.

20. Friedman SE, Andrus BW. Obesity and pulmonary hypertension: a review of pathophysiologic mechanisms. J Obes 2012; 2012:505274.

21. Chan AL, Juarez MM, Shelton DK, MacDonald T, Li CS, Lin TC, et al. Novel computed tomographic chest metrics to detect pulmonary hypertension. BMC Med Imaging. 2011 29;11:7

22. Lavie CJ, Milani RV, Ventura HO. Obesity and cardiovascular disease: risk factor, paradox, and impact of weight loss. J Am Coll Cardiol 2009; 53(21):1925-32.

23. Mahabadi AA, Massaro JM, Rosito GA, Levy D, Murabito JM, Wolf PA, et al. Association of pericardial fat, intrathoracic fat, and visceral abdominal fat with cardiovascular disease burden: the Framingham Heart Study. Eur Heart J. 2009;30(7):850-6.

24. Owan T, Litwin SE. Is there a cardiomyopathy of obesity? Curr Heart Fail Rep 2007; 4(4):221-8.

25. Murphy ML, Blue LR, Thenabadu PN, Phillips JR, Ferris EJ. The reliability of the routine chest roentgenogram for determination of heart size based on specific ventricular chamber evaluation at postmortem. Invest Radiol 1985; 20(1):21-5. 
26. Stary HC. The sequence of cell and matrix changes in atherosclerotic lesions of coronary arteries in the first forty years of life. Eur Heart J 1990; 11: 3-19.

27. Abedin M, Tintut Y, Demer LL. Vascular calcification: mechanisms and clinical ramifications. Arterioscler Thromb Vasc Biol 2004; 24(7):1161-70.

28. Iribarren C, Sidney S, Sternfeld B, Browner WS. Calcification of the aortic arch: risk factors and association with coronary heart disease, stroke, and peripheral vascular disease. JAMA 2000;283(21):28105 .

29. Shuster A, Patlas M, Pinthus JH, Mourtzakis M. The clinical importance of visceral adiposity: a critical review of methods for visceral adipose tissue analysis. Br J Radiol 2012; 85(1009):1-10.

30. Rosito GA, Massaro JM, Hoffmann U, Ruberg FL, Mahabadi AA, Vasan RS, et al. Pericardial fat, visceral abdominal fat, cardiovascular disease risk factors, and vascular calcification in a community-based sample: the Framingham Heart Study. Circulation. 2008 5;117(5):605-13.

31. McLaughlin T, Lamendola C, Liu A, Abbasi F. Preferential fat deposition in subcutaneous versus visceral depots is associated with insulin sensitivity. J Clin Endocrinol Metab. 2011 ;96(11):1756-60.

32. Dahlén EM, Bjarnegård N, Länne T, Nystrom FH, Ostgren CJ. Sagittal abdominal diameter is a more independent measure compared with waist circumference to predict arterial stiffness in subjects with type 2 diabetes - a prospective observational cohort study. Cardiovasc Diabetol 2013; 12:55.

33. Budoff MJ, Achenbach S, Blumenthal RS, Carr JJ, Goldin JG, Greenland P, et al. American Heart Association Committee on Cardiovascu- lar Imaging and Intervention; American Heart Association Council on Cardiovascular Radiology and Intervention; American Heart Association Committee on Cardiac Imaging, Council on Clinical Cardiology. Assess- ment of coronary artery disease by cardiac computed tomography: a scientific statement from the American Heart Association Committee on Cardiovascular Imaging and Intervention, Council on Cardiovascular Radiology and Intervention, and Committee on Cardiac Imaging, Council on Clinical Cardiology. Circulation 2006; 114(16):1761-91.

\section{Figure Legends}

Fig. 1. 45 year-old man with pneumonia. Unenhanced thorax $\mathrm{CT}$ image represents thin linear fatty infiltration in submucosal layer of gastric wall with attenuation value $<-10 \mathrm{HU}$.

Fig. 2. 43 year-old man with cough complaint. The measurements of the ascending aorta, the descending aorta, the main pulmonary artery, and the right and left pulmonary arteries were recorded in the axial plane at the level of the pulmonary artery bifurcation.

Fig. 3. 51 year-old man with solitary pulmonary nodule. The measurements of the long and short cardiac axes and the maximum transverse thoracic diameter were obtained at the axial slice level that represented the maximum size of the heart.

Fig. 4. 59-year-old man with gastric wall fat halo sign.

A, Unenhanced sagittal CT image shows extensive calcific atherosclerotic plaques in aorta

B, Unenhanced axial CT image shows extensive calcific atherosclerotic plaques in coronary arteries

\section{Hosted file}

Tables.pdf available at https://authorea.com/users/383097/articles/499003-gastric-wall-fatfalo-sign-as-a-risk-factor-for-cardiovascular-diseases 

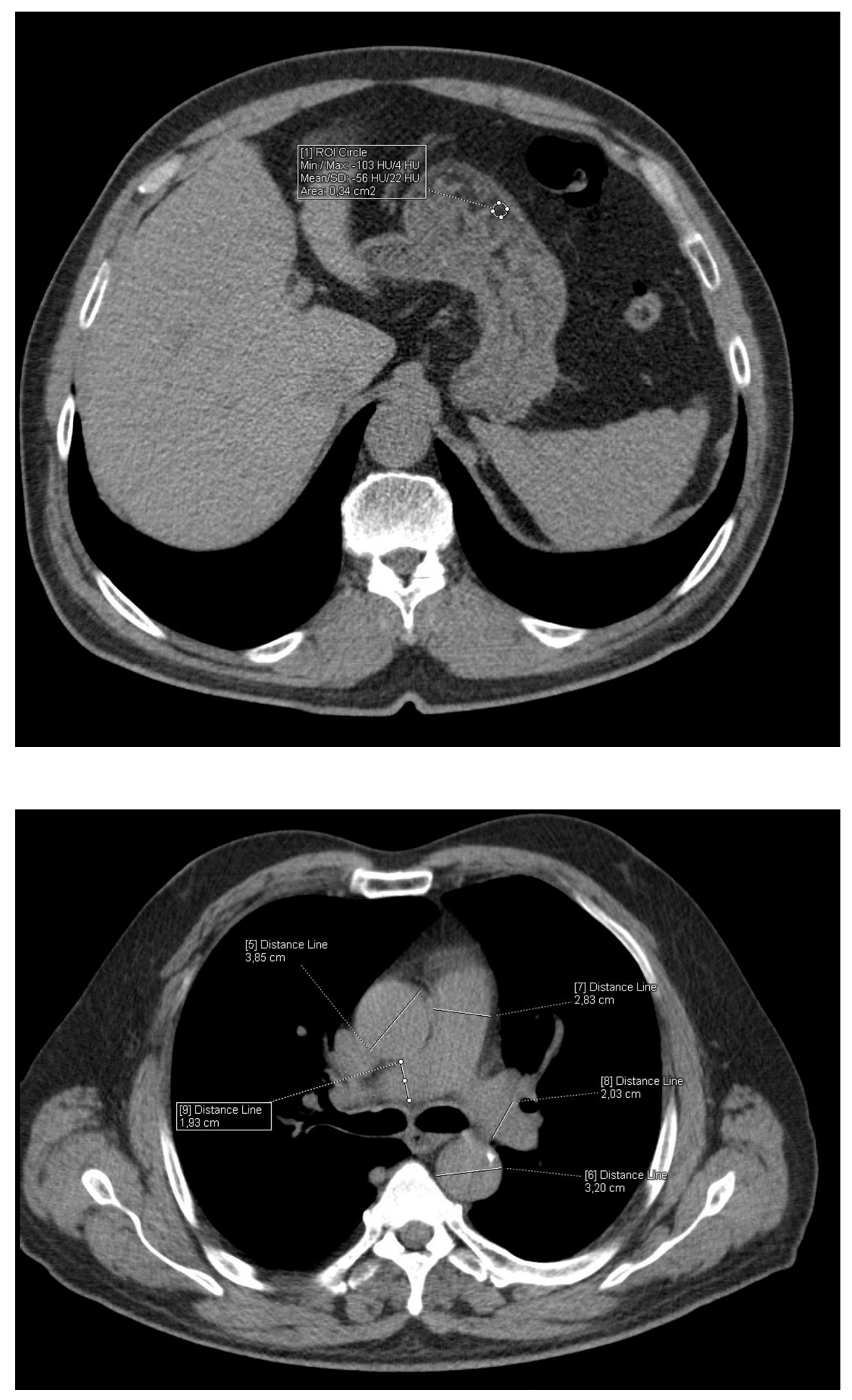


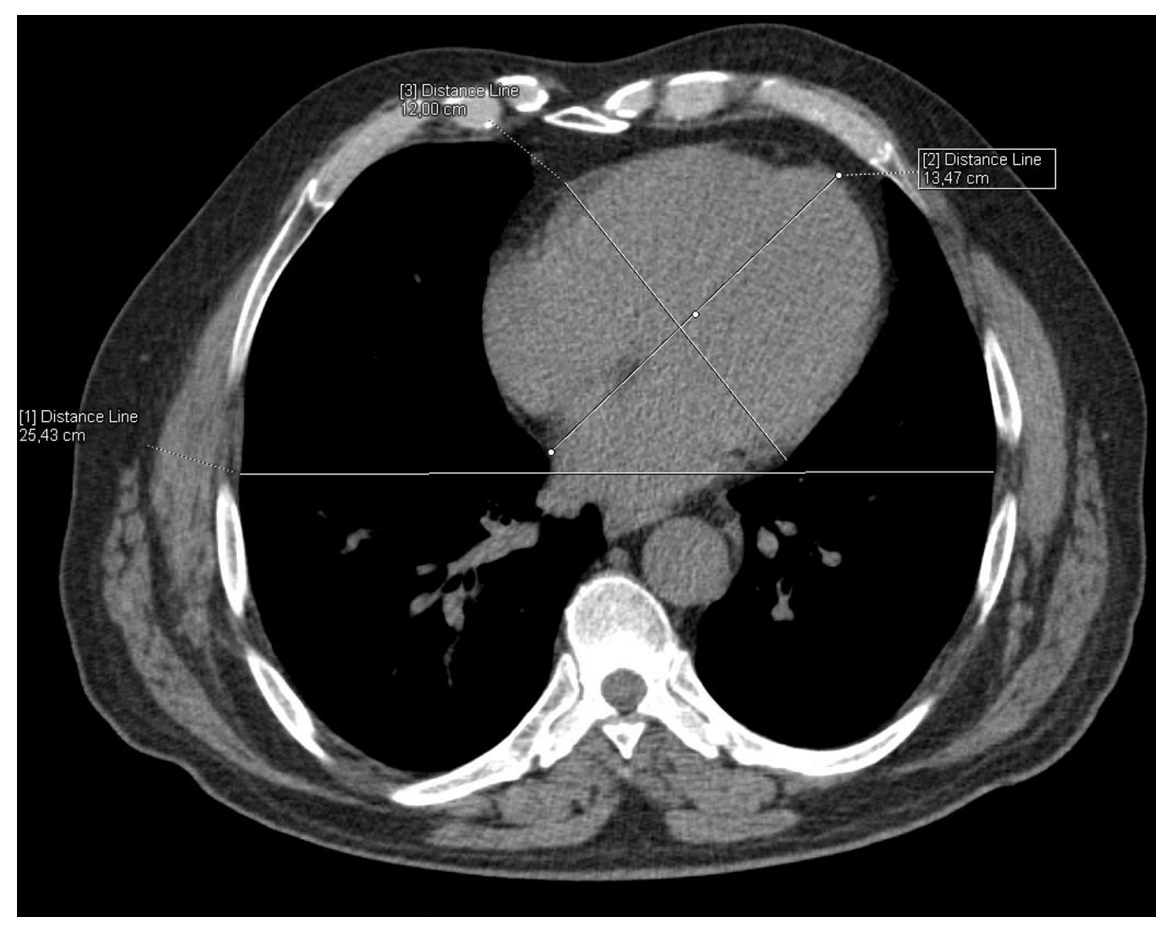




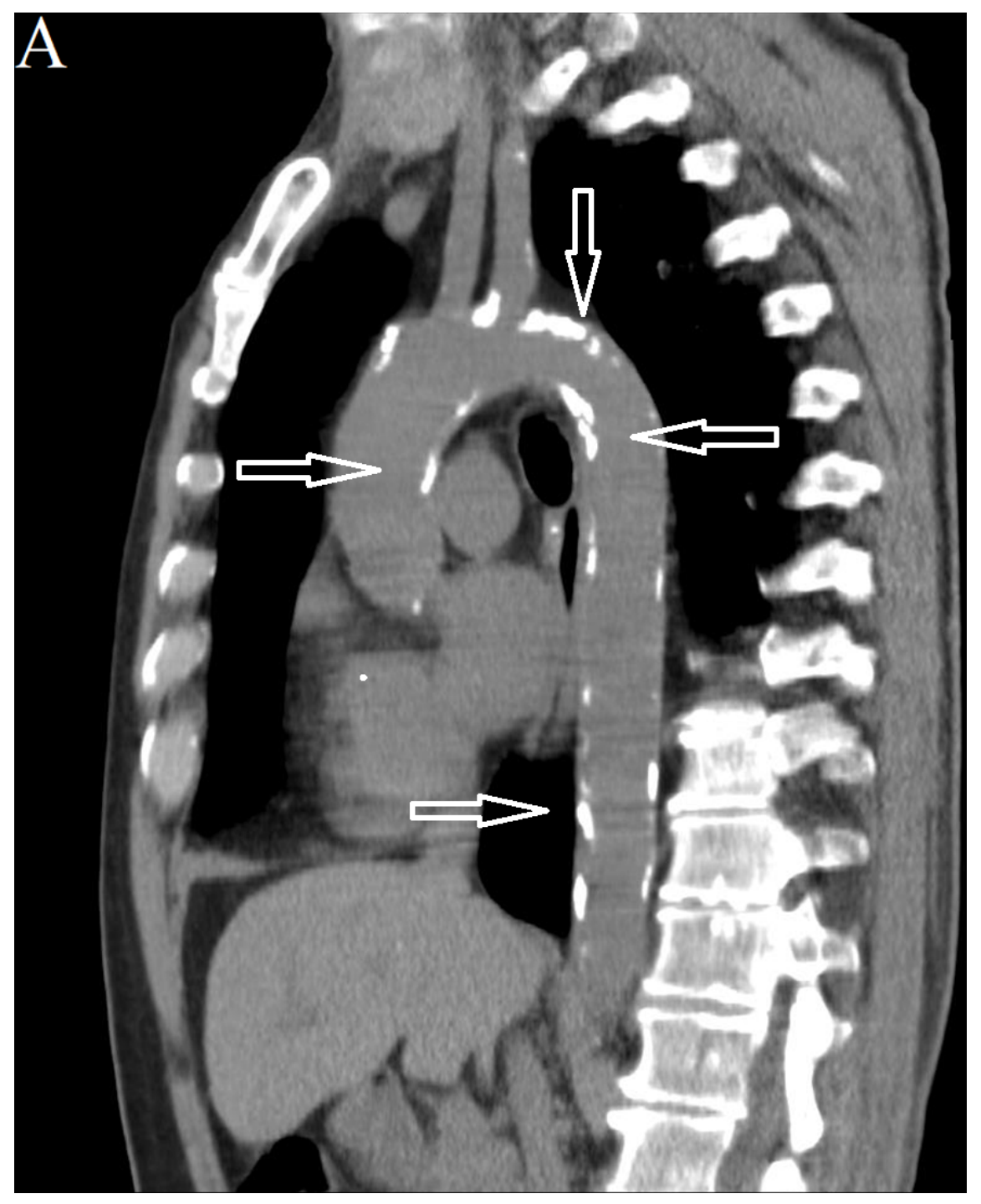




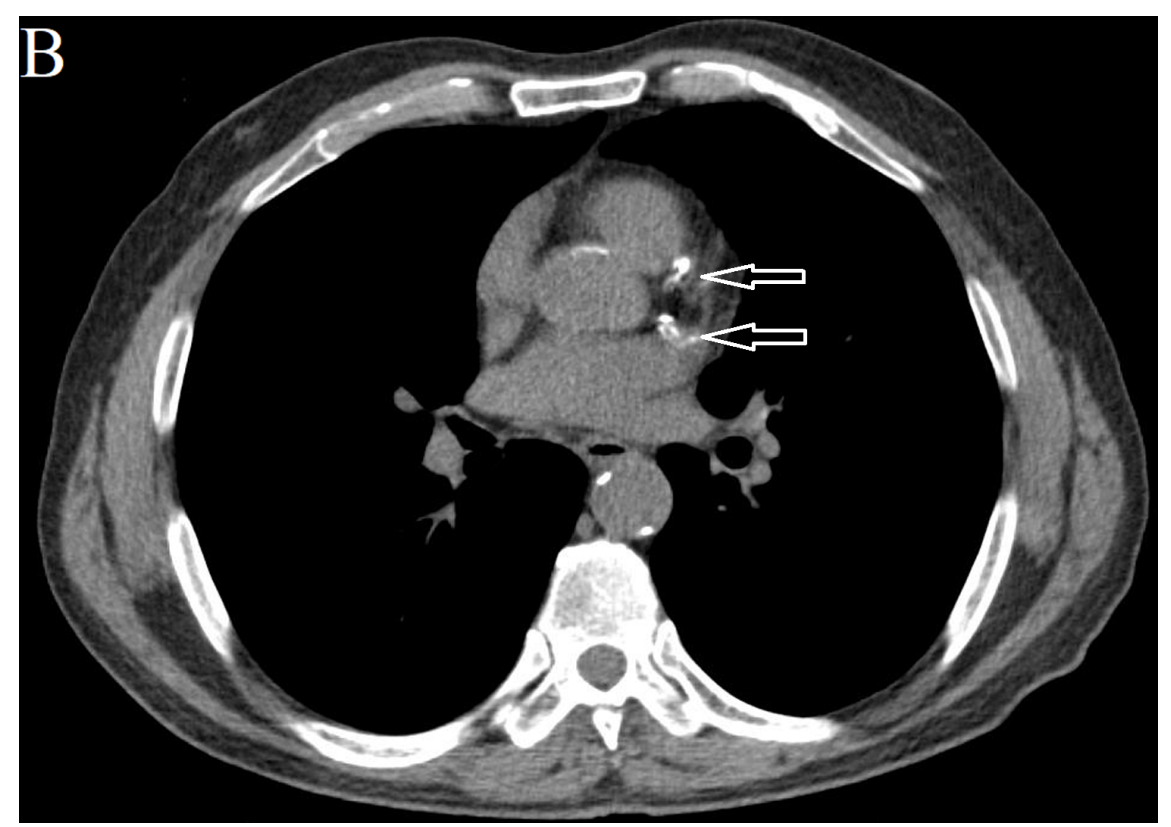

